\title{
Correction to: Blocking of opioid receptors in experimental formaline- inactivated respiratory syncytial virus (FI-RSV) immunopathogenesis: from beneficial to harmful impacts
}

\author{
Vahid Salimi ${ }^{1}$ - Habib Mirzaei ${ }^{1}$ Ali Ramezani ${ }^{1}$. Alireza Tahamtan ${ }^{1,2} \cdot$ Abbas Jamali $^{3}$. Shahram Shahabi ${ }^{4}$. \\ Maryam Golara ${ }^{5} \cdot$ Bagher Minaei $^{6} \cdot$ Mohammad Javad Gharagozlou $^{7} \cdot$ Mahmood Mahmoodi $^{8} \cdot$ Louis Bont $^{9}$. \\ Fazel Shokri ${ }^{5}$. Talat Mokhtari-Azad ${ }^{1}$
}

Published online: 22 September 2018

c) Springer-Verlag GmbH Germany, part of Springer Nature 2018

\section{Correction to: \\ Medical Microbiology and Immunology \\ (2018) 207:105-115 \\ https://doi.org/10.1007/s00430-017-0531-0}

In the original publication, seventh author's name was incorrectly published as 'Maryam Golaram'. The correct name should read as 'Maryam Golara'.

The original article can be found online at https://doi.org/10.1007/ s00430-017-0531-0.

Talat Mokhtari-Azad

mokhtari@sina.tums.ac.ir

1 Department of Virology, School of Public Health, Tehran University of Medical Sciences, Tehran, Iran

2 Department of Microbiology, School of Medicine, Golestan University of Medical Sciences, Gorgan, Iran

3 Influenza and Other Respiratory Viruses Department, Pasteur Institute of Iran, Tehran, Iran

4 Cellular and Molecular Research Center, Urmia University of Medical Sciences, Urmia, Iran

5 Department of Immunology, School of Public Health, Tehran University of Medical Sciences, Tehran, Iran

6 Department of Anatomy, Faculty of Medicine, Tehran University of Medical Sciences, Tehran, Iran

7 Department of Pathology, Faculty of Veterinary Medicine, University of Tehran, Tehran, Iran

8 Epidemiology and Biostatistics Department, School of Public Health, Tehran University of Medical Sciences, Tehran, Iran

9 Department of Paediatrics, Wilhelmina Children's Hospital, University Medical Centre Utrecht, Utrecht, The Netherlands 